\title{
A novel study design for antibiotic trials in acute exacerbations of COPD: MAESTRAL methodology
}

This article was published in the following Dove Press journal:

International Journal of COPD

28 June 2011

Number of times this article has been viewed

\section{Robert Wilson' \\ Antonio Anzueto ${ }^{2}$ \\ Marc Miravitlles ${ }^{3}$ \\ Pierre Arvis ${ }^{4}$ \\ Geneviève Faragó ${ }^{5}$ \\ Daniel Haverstock ${ }^{6}$ \\ Mila Trajanovic ${ }^{5}$ \\ Sanjay Sethi ${ }^{7}$}

'Host Defence Unit, Royal Brompton Hospital, London, England, UK;

${ }^{2}$ University of Texas Health Science

Center at San Antonio, South

Texas Veterans HealthCare System,

San Antonio, TX, USA; ${ }^{3}$ Institut

d'Investigacions Biomèdiques

August Pi I Sunyer (IDIBAPS), Ciber

de Enfermedades Respiratorias

(CIBERES), Hospital Clinic, Barcelona,

Spain; ${ }^{4}$ Bayer HealthCare, Loos,

France; ${ }^{5}$ Bayer Inc, Toronto, ON,

Canada; ${ }^{6}$ Bayer HealthCare

Pharmaceuticals, Montville, NJ, USA;

${ }^{7}$ Division of Pulmonary, Critical Care and Sleep Medicine, University at Buffalo, State University of New York, Buffalo, NY, USA
Correspondence: Robert Wilson Host Defence Unit, Royal Brompton Hospital, Sydney Street, London SW3 6NP, UK

Tel +4420735I 8337

Fax +4420735I 8338

Email r.wilson@rbht.nhs.uk

\begin{abstract}
Antibiotics, along with oral corticosteroids, are standard treatments for acute exacerbations of chronic obstructive pulmonary disease (AECOPD). The ultimate aims of treatment are to minimize the impact of the current exacerbation, and by ensuring complete resolution, reduce the risk of relapse. In the absence of superiority studies of antibiotics in AECOPD, evidence of the relative efficacy of different drugs is lacking, and so it is difficult for physicians to select the most effective antibiotic. This paper describes the protocol and rationale for MAESTRAL (moxifloxacin in AECBs [acute exacerbation of chronic bronchitis] trial; www.clinicaltrials.gov: NCT00656747), one of the first antibiotic comparator trials designed to show superiority of one antibiotic over another in AECOPD. It is a prospective, multinational, multicenter, randomized, double-blind controlled study of moxifloxacin (400 mg PO [per os] once daily for 5 days) vs amoxicillin/clavulanic acid (875/125 mg PO twice daily for 7 days) in outpatients with COPD and chronic bronchitis suffering from an exacerbation. MAESTRAL uses an innovative primary endpoint of clinical failure: the requirement for additional or alternate treatment for the exacerbation at 8 weeks after the end of antibiotic therapy, powered for superiority. Patients enrolled are those at high-risk of treatment failure, and all are experiencing an Anthonisen type I exacerbation. Patients are stratified according to oral corticosteroid use to control their effect across antibiotic treatment arms. Secondary endpoints include quality of life, symptom assessments and health care resource use.
\end{abstract}

Keywords: AECOPD, moxifloxacin, amoxicillin/clavulanic acid, clinical trial design, exacerbation, antibiotic

\section{Introduction}

Acute exacerbations of chronic obstructive pulmonary disease (AECOPD), which is usually associated with chronic bronchitis, drive progressive lung function decline in patients, ${ }^{1-3}$ are major contributors to mortality, morbidity and reductions in quality of life, ${ }^{4-9}$ and the resulting hospitalizations markedly increase health care costs. ${ }^{7,10}$ The mainstays of AECOPD treatment are intensification or addition of short-acting bronchodilators, and in selected patients, antibiotics with or without systemic corticosteroids. ${ }^{5,11}$ However, treatment failure rates in many recent antibiotic studies are high, which could be related to inadequate antibiotic efficacy. ${ }^{12,13}$ Incomplete resolution of the initial exacerbation may also influence the risk of relapse: many patients experience a further exacerbation within a few weeks, ${ }^{13,14}$ with relapse rates varying between antibiotics. ${ }^{14}$ Therefore, it is likely that improving clinical outcomes requires the use of the most effective antibiotic available.

Proof of superiority of one antibiotic over another in AECOPD is lacking, ${ }^{5,11}$ as are high-quality clinical data to inform clinical guidelines. ${ }^{15,16}$ Although there are many comparative studies of different antibiotics in AECOPD, no single drug has yet shown superiority for the primary endpoint in a clinical trial. This lack of evidence of 
superiority is in part because many studies were powered only for noninferiority, but may also be due to issues of patient selection (patient characteristics predating the current exacerbation can negatively affect outcomes) and endpoint selection in clinical trials. ${ }^{11,15,17,18}$

There is a clear unmet need for superiority trials in AECOPD,${ }^{19}$ in particular between antibiotics commonly used in AECOPD and in patients at high risk of poor clinical outcomes who are most likely to benefit from the most effective therapies. ${ }^{11,18,19}$ While trials of antibiotics in AECOPD have yet to show superiority for the primary endpoint, some have demonstrated superiority for secondary endpoints, the most recent of which was the MOSAIC study. ${ }^{20}$ This compared moxifloxacin and a basket of comparators (amoxicillin/clarithromycin/cefuroxime axetil) in patients with Anthonisen type I exacerbations ${ }^{21}$ and mild-to-moderate COPD. In MOSAIC, moxifloxacin was superior to comparator antibiotics for clinical cure (defined as a return to baseline health status) and bacterial eradication. Longer-term outcomes from MOSAIC showed that moxifloxacin was also superior to comparators in terms of lower relapse rates. ${ }^{20}$

This paper describes the MAESTRAL (moxifloxacin in AECBs [acute exacerbation of chronic bronchitis] trial; clinical trial.gov number: NCT00656747) study design and the rationale for its novel features. These include powering the study for superiority testing, selection of chronic bronchitis patients with moderate-to-severe COPD and assessment of the utility of an 8-week clinical endpoint.

\section{MAESTRAL methodology Study design}

MAESTRAL is a prospective, multinational, multicenter, randomized, double-blind, double-dummy controlled study. The primary objective of MAESTRAL is to compare the efficacy of a 5-day course of moxifloxacin to that of a 7-day course of amoxicillin/clavulanic acid in the treatment of outpatients experiencing AECOPD who are at high risk of treatment failure. The study is powered to show clinical superiority between antibiotic treatments. It uses an innovative clinical endpoint of clinical failure rates over an 8-week period after treatment with an antibiotic. The study design is shown in Figure 1.

\section{Study population \\ Inclusion and exclusion criteria}

Outpatients with COPD and chronic bronchitis suffering from an exacerbation are enrolled in the MAESTRAL study.

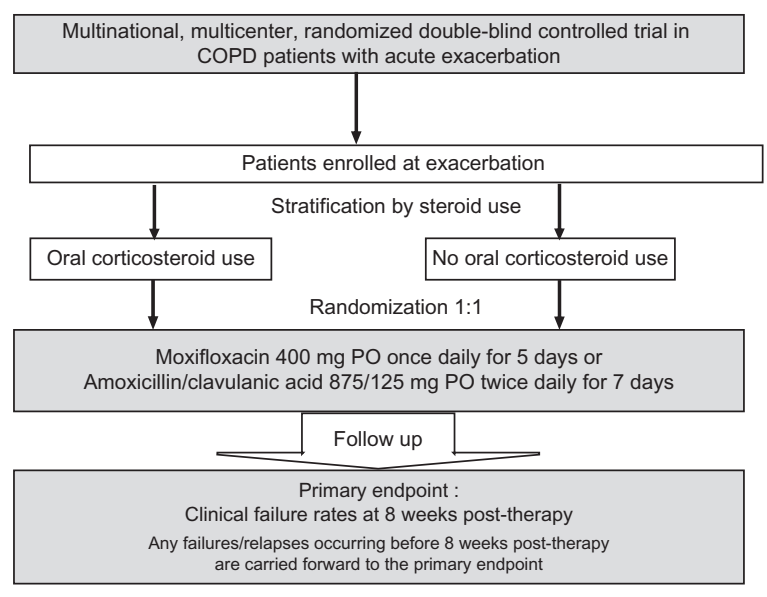

Figure I MAESTRAL study design.

Patients must be $\geq 60$ years old, have a documented history of $\geq 2$ exacerbations within the previous year requiring a course of systemic antibiotics and/or systemic corticosteroids, postbronchodilator forced expiratory volume in 1 second $\left(\mathrm{FEV}_{1}\right) \leq 60 \%$ predicted, $\mathrm{FEV}_{1} /$ forced vital capacity $(\mathrm{FVC})<70 \%$, and be current or past cigarette smokers with a $\geq 20$ pack-year smoking history. They must also be experiencing an Anthonisen type I exacerbation (the presence of all three of purulent sputum, increased sputum volume, and increased dyspnea ${ }^{21}$ as confirmed by the investigator) and be suitable for treatment with oral antibiotics. Patients must also be exacerbation-free for at least 30 days prior to enrollment.

The main exclusion criteria are contraindications to study drugs, immunosuppression, structural lung diseases (for example, known diffuse bronchiectasis), and systemic antibiotics or corticosteroids within 30 days prior to enrollment. The full list of exclusion criteria is given in Supplementary Section 1.

\section{Stratification by steroid use}

To ensure an even distribution between treatment arms of patients receiving corticosteroids, patients are stratified according to the coadministration of a short course (5 days) of systemic corticosteroid therapy (30-40 mg/day of prednisolone or equivalent) for the current exacerbation, where the first dose is administered on the same or next day as the study drug (Figure 1 and Supplementary Section 2). Following the end of this 5-day course of steroid treatment, down-titration for up to 5 days is allowed. Agents used to manage the ongoing COPD (long-acting bronchodilators and inhaled corticosteroids) will be maintained on a stable dose and regimen during treatment and for the 8 weeks of follow-up. The choice 
of steroids is at the treating physician's discretion within guidelines set out in the study protocol. All comedications will be recorded for the duration of the study.

\section{Ethical approval}

All patients must provide written informed consent, according to the International Conference on Harmonisation Good Clinical Practice Guidelines and local legal requirements. The study is being carried out according to Good Clinical Practice Guidelines and according to the principles of the Declaration of Helsinki. Documented approval has been obtained from the appropriate ethical committees and Institutional Review Boards.

\section{Microbiology}

Spontaneous sputum samples are to be obtained from all patients, assessed in a local laboratory, and examined for the presence of polymorphonuclear cells, squamous epithelial cells, and bacteria. All potentially pathogenic bacteria (PPB) are to be identified (Streptococcus pneumoniae, Moraxella catarrhalis, Pseudomonas aeruginosa, Haemophilus spp., Enterobacteriaceae spp., and Staphylococcus aureus) and pure subcultures frozen and subsequently forwarded to a central microbiology laboratory. There, they will be re-identified and minimum inhibitory concentrations (MICs) determined for moxifloxacin and amoxicillin/clavulanic acid and a range of other antibiotics by the reference Clinical Laboratory Standards Institute broth microdilution method. ${ }^{22}$ MICs for penicillin are determined for $S$. pneumoniae, and meticillin resistance is determined in $S$. aureus.

\section{Antibiotic treatments}

Patients receive either moxifloxacin (Bayer Schering Pharma, Wuppertal, Leverkusen or Berlin, Germany) $400 \mathrm{mg}$ PO once daily for 5 days or amoxicillin/clavulanic acid (STADApharm GmbH, Bad Vilbel, Germany) 875/125 mg PO twice daily for 7 days. Moxifloxacin and amoxicillin/clavulanic acid are well established as effective therapies for exacerbations of COPD and chronic bronchitis with good clinical and bacteriological efficacy in AECOPD. ${ }^{23-25}$ They are also both included within guidelines as recommended therapies for patients with moderate-to-severe exacerbations. ${ }^{15,16}$ The dose chosen for both drugs is that recommended in European guidelines ${ }^{15}$ and is in common use in the regions where MAESTRAL is being carried out. The pharmacokinetic/ pharmacodynamic (PK/PD) properties of both drugs suggest that they are an adequate treatment for $S$. pneumoniae isolates with an MIC $\leq 2 \mathrm{mg} / \mathrm{L} .{ }^{26}$ Importantly, the dose of amoxicillin/clavulanic acid chosen (875/125 mg PO twice daily for 7 days) has a comparable clinical efficacy and safety profile to a higher dose, shorter course of long-acting amoxicillin/clavulanic acid (2000/125 mg PO twice daily for 5 days). ${ }^{24}$

\section{Clinical and bacteriological endpoints}

The primary efficacy endpoint of MAESTRAL is clinical failure at the 8 -weeks post-therapy visit. Clinical failure is defined as the requirement for additional or alternate treatment with systemic antibiotics and/or systemic corticosteroids (including increased dose or duration of treatment), and/or hospitalization within 8 weeks post-therapy for an exacerbation of respiratory symptoms. Clinical failures occurring during therapy or follow-up are carried forward until 8 weeks post-therapy. Clinical failures occurring during therapy or follow-up are carried forward until 8 weeks post-therapy.

A range of secondary endpoints is also defined; the full list is given in Table 1. These include clinical response rates at interim time points and bacteriological outcome at all time points, lung function tests, medication use, and quality of life (St George's Respiratory Questionnaire [SGRQ]) ${ }^{27}$ and symptom assessments (Acute Exacerbation of Chronic Bronchitis Symptom Scale [AECB-SS]). ${ }^{28,29}$

\section{Clinical and bacteriological efficacy assessments}

The study consists of the following visits: enrollment/ randomization (day 1 is the first day of study drug treatment), during therapy (treatment day $4 \pm 1$ ), end of therapy (day $13 \pm 1$ after start of study drug treatment), 4 weeks post-therapy (day $35 \pm 3$ after start of study drug treatment), and 8 weeks post-therapy (day $63 \pm 3$ after start of study drug treatment). Data collected at each visit are shown in Table 2.

Assessment of clinical response to therapy is based on determination of the effect of therapy on auscultatory findings, chest pain/discomfort, cough frequency, dyspnea, sputum purulence, sputum consistency, and sputum volume. Clinical response is assessed as: clinical improvement (during therapy only), clinical failure, indeterminate, clinical cure, continued clinical cure, and clinical relapse (definitions are given in Supplementary Section 3). Clinical failure rates can be assessed during nonprotocol-defined visits as well as during therapy, at end of therapy, and at the 4 and 8 -week post-therapy visits. Premature discontinuations due to study 
Table I Secondary endpoints

\begin{tabular}{|c|c|}
\hline Category & Endpoint \\
\hline Clinical failure measures & $\begin{array}{l}\text { - Clinical failure rates during therapy, at end of therapy and at } 4 \text { weeks post-therapy } \\
\text { - Clinical failure rates (for patients with positive sputum culture at enrollment) during therapy, at end } \\
\text { of therapy, and at } 4 \text { and } 8 \text { weeks post-therapy } \\
\text { - Clinical failure rates for patients with coadministration of systemic corticosteroids } \\
\text { - Clinical failure rates for patients without coadministration of systemic corticosteroids }\end{array}$ \\
\hline Bacteriological outcomes & - Bacteriological eradication rates during therapy, at end of therapy, and at 4 and 8 weeks post-therapy \\
\hline Symptom burden and quality of life & $\begin{array}{l}\text { - Weekly mean symptom scores measured by the AECB-SS } \\
\text { - Rates and speed of symptom relief measured by the AECB-SS } \\
\text { - Changes in symptom burden measured by the AECB-SS } \\
\text { - Changes in health-related quality of life measured by the SGRQ } \\
\text { - Lung function (spirometry): between-group comparisons at each assessment visit }\end{array}$ \\
\hline $\begin{array}{l}\text { Comedications and health care } \\
\text { resource use }\end{array}$ & $\begin{array}{l}\text { - Need for any change in dosage or additional respiratory medication such as bronchodilators and } \\
\text { inhaled corticosteroids, but excluding short-acting bronchodilators } \\
\text { - Health care resource use/consumption related to COPD/chronic bronchitis management including } \\
\text { rescue medications, concomitant medications, therapeutic adjuncts, diagnostic procedures, other } \\
\text { medical care/medical staff requirements, hospitalizations, and work productivity and activity } \\
\text { impairment }\end{array}$ \\
\hline Safety & $\begin{array}{l}\text { - Safety and tolerability of moxifloxacin versus amoxicillin/clavulanic acid, with particular attention to } \\
\text { rates of diarrhea }\end{array}$ \\
\hline
\end{tabular}

Abbreviations: AECB-SS, Acute Exacerbation of Chronic Bronchitis Symptom Scale; COPD, chronic obstructive pulmonary disease; SGRQ, St George's Respiratory Questionnaire.

drug failure will also be treated as failures. For patients who discontinue study drug for reasons other than efficacy, the assessment at 8-week post-therapy visit will be used where completed. For those patients who do not return for the 8 -week post-therapy visit, the response will be considered missing. These patients will be excluded from the per protocol population as will those with an indeterminate clinical outcome at the last available assessment.

The categories of bacteriological response to therapy are: eradication without superinfection or reinfection, presumed eradication, persistence, presumed persistence, eradication with superinfection, eradication with reinfection, eradication with recurrence, continued eradication, continued presumed eradication, and indeterminate (see Supplementary Section 3). Bacteriological response rates are assessed during therapy, at end of therapy and at 4 and 8 weeks post-therapy.

\section{Data Review Committee}

There has been some concern in previous antibiotic studies regarding inconsistent assessment of clinical outcomes by treating physicians. To validate the physician's assessment of clinical outcomes an independent Data Review Committee (DRC) will assess the data for all clinical failures and indeterminate assessments prior to the unblinding of the treatment assignment of datasets. Their objective is to confirm the primary clinical outcome and to validate the inclusion of the patient's data in the primary analysis.

\section{Safety assessments}

The safety of treatment is monitored by clinical observations at each visit following enrollment. All adverse events (AEs) are recorded up to 8 weeks post-therapy. All AEs are assessed in terms of their severity, intensity (mild, moderate or severe), and relationship to the study drug. Investigators are asked to monitor any patients with diarrhea, and if Clostridium difficile-related diarrhea is suspected, investigators are asked to take appropriate clinical measures, including $C$. difficile toxin detection and remedial therapy where warranted.

\section{Analyses Efficacy populations}

The primary population for assessment of clinical outcomes is the per protocol (PP) population. These patients have all had an acute exacerbation at enrollment and have received the study drug for a minimum of 48 hours (cases of clinical failure) or received $\geq 80 \%$ of study medication (cases of clinical cure). All have data for clinical evaluation at 8 weeks post-therapy (except for clinical failures prior to the 8 weeks post-therapy visit) and have no protocol violations. The intent-to-treat (ITT)/safety population includes all patients randomized who received at least one dose of study drug and with one observation after initiation of study treatment. The microbiologically valid population is drawn from the PP population and comprises all patients with at least one PPB cultured from sputum provided prior to start of therapy and where a bacteriological evaluation is available during 
Table 2 Data collected at each study visit

\begin{tabular}{|c|c|c|c|c|c|}
\hline \multirow[t]{2}{*}{ Assessment } & \multicolumn{5}{|c|}{ Visit } \\
\hline & $\begin{array}{l}\text { Enrollment/ } \\
\text { randomization } \\
\text { (day } I \text { is the first day } \\
\text { of treatment) }\end{array}$ & $\begin{array}{l}\text { During therapy } \\
\text { (treatment day } \\
4 \pm \text { I day) }\end{array}$ & $\begin{array}{l}\text { End of therapy } \\
\text { (day } 13 \pm I \text { day } \\
\text { after start of } \\
\text { treatment) }\end{array}$ & $\begin{array}{l}\text { Four weeks } \\
\text { post-therapy } \\
\text { (day } 35 \pm 3 \text { days after } \\
\text { start of treatment) }\end{array}$ & $\begin{array}{l}\text { Eight weeks } \\
\text { post-therapy } \\
\text { (day } 63 \pm 3 \text { days after } \\
\text { start of treatment) }\end{array}$ \\
\hline $\begin{array}{l}\text { Sputum specimens } \\
\text { for bacteriological } \\
\text { examination (macroscopic } \\
\text { assessment of purulence, } \\
\text { microscopic evaluations, } \\
\text { and culture) }\end{array}$ & $\checkmark$ & $\checkmark$ & $\checkmark$ & $\checkmark$ & $\checkmark$ \\
\hline Clinical response $\mathrm{e}^{\mathrm{a}}$ & - & $\checkmark$ & $\checkmark$ & $\checkmark$ & $\checkmark$ \\
\hline Spirometry & $\checkmark$ & $\checkmark$ & $\checkmark$ & $\checkmark$ & $\checkmark$ \\
\hline $\begin{array}{l}\text { AECB-SS questionnaire } \\
\text { (daily from start of } \\
\text { treatment to end } \\
\text { of therapy visit and } \\
\text { at post-therapy visits) }\end{array}$ & $\checkmark$ & $\checkmark$ & $\checkmark$ & - & - \\
\hline $\begin{array}{l}\text { SGRQ (self-administered } \\
\text { at each visit) }\end{array}$ & $\checkmark$ & - & $\checkmark$ & $\checkmark$ & $\checkmark$ \\
\hline $\begin{array}{l}\text { Health care resource } \\
\text { consumption related to } \\
\text { AECOPD/COPD (all } \\
\text { resource use recorded) }\end{array}$ & $\checkmark$ & $\checkmark$ & $\checkmark$ & $\checkmark$ & $\checkmark$ \\
\hline $\begin{array}{l}\text { Adverse events, } \\
\text { concomitant medications } \\
\text { (recorded at each visit) }\end{array}$ & $\checkmark$ & $\checkmark$ & $\checkmark$ & $\checkmark$ & $\checkmark$ \\
\hline $\begin{array}{l}\text { Changes in respiratory } \\
\text { symptoms }\end{array}$ & - & $\checkmark$ & $\checkmark$ & $\checkmark$ & $\checkmark$ \\
\hline
\end{tabular}

Notes: alf the subject did not improve during therapy, or experienced a relapse, an unscheduled clinic visit (premature discontinuation) was booked and the subject underwent all 8 weeks post-therapy visit evaluations prior to initiation of an additional or alternative treatment; if the investigator assessment was clinical failure, the subject was prematurely terminated from the study and was not followed further.

Abbreviations: AECB-SS, Acute Exacerbation of Chronic Bronchitis Symptom Scale; SGRQ, St George's Respiratory Questionnaire.

the study. The ITT with causative organisms population includes patients valid for ITT with at least one pretherapy PPB.

\section{Statistics}

\section{Sample size calculation}

To power MAESTRAL for superiority, the objective is to ensure that 540 valid PP patients are enrolled in each treatment arm; therefore, at least 1350 COPD patients with an Anthonisen type I exacerbation ${ }^{21}$ are required to be randomized. Patients will be drawn from across 30 countries. This high number of patients is driven by a $6 \%$ margin - a more rigorous definition of failure rates than the standard $10 \%$ normally used in noninferiority trials. ${ }^{30}$

\section{Primary analysis}

The primary aim of the study is to show noninferiority (defined as a difference in failure rates of $\leq 6 \%$ using a one-sided test at a level of $2.5 \%$ ) of moxifloxacin vs amoxicillin/clavulanic acid in the PP population. If noninferiority is statistically proven, the possibility that moxifloxacin is superior to amoxicillin/clavulanic acid will be tested in the ITT population, using a one-sided test at the $2.5 \%$ level. The primary ITT analysis will be clinical failure vs all other evaluations (clinical cure, indeterminate, and missing). Two sensitivity analyses are planned: (1) missing responses will be combined with indeterminates and clinical failures in an overall nonsuccess category, and (2) missing and indeterminate responses will be excluded and only cures or failures will be considered. The 95\% two-sided confidence interval (CI) of the difference of two clinical failure rates (treatment group 'moxifloxacin' minus treatment group 'amoxicillin/clavulanic acid') will be calculated using Mantel-Haenszel weights for the strata (geographic region and stratification by corticosteroid use, as outlined in Supplementary Section 2). If the upper limit of the $\mathrm{CI}$ is $<6 \%$, it will prove that treatment with moxifloxacin is clinically no less effective than treatment with amoxicillin/ 
clavulanic acid. If the upper limit of this $\mathrm{CI}$ is $<0$, superiority of treatment with moxifloxacin will be proven. To check the appropriateness of the calculation of the Mantel-Haenszel weighted CIs, the Breslow-Day test, or Zelen's test, will be performed to test for homogeneity of odds ratios across strata. If the test indicates a treatment-by-stratum interaction, exploratory analyses will be performed to find the source of this interaction. If such a source is found, the MantelHaenszel weighted CI will be calculated using weighting based on variables explaining the interaction.

Demographic and baseline characteristics will be summarized by treatment group for the PP and ITT populations and compared using the Cochran-Mantel-Haenszel test adjusted for strata and region.

Secondary clinical and bacteriological endpoints will be analyzed as for the primary analysis, but using appropriate patient populations and time points. Clinical and bacteriological outcomes at a variety of time points (during therapy, end of therapy, 4 weeks post-therapy, and 8 weeks post-therapy) will be investigated in subpopulations of special interest, such as those with and without coadministration of systemic corticosteroids. The full list of subpopulations of interest is given in Supplementary Section 4.

AECB-SS and SGRQ scores will be determined and analyzed separately. The main analysis for the SGRQ data will compare the mean change from baseline in the total SGRQ score between treatment groups 8 weeks after the end of therapy. For AECB-SS, the means, standard deviations, medians, minimums, and maximums will be provided for the total score and change from baseline in total score at each visit, by treatment group. For health care resource use, if the homogeneity of between-country resource consumption is satisfactory, the data will be pooled, summarized, and $95 \%$ CIs estimated.

For the safety analysis, a Cochran-Mantel-Haenszel test will assess the difference in incidence rates between treatment groups for the overall rate of premature discontinuations, discontinuations due to AEs, and discontinuations due to insufficient therapeutic effect. Other safety data will be tabulated; vital signs will be analyzed descriptively.

\section{Discussion}

Clinical trials of antibiotics in AECOPD have traditionally been short-term, included relatively small numbers of patients, and focused largely on the cure of an existing exacerbation. Most of these trials have been powered to show noninferiority, making it difficult for physicians to select the 'best' antibiotic for a particular patient. ${ }^{11,31,32}$ MAESTRAL aims to address all these issues by recruiting sufficient patients ( $\mathrm{n} \geq 1350$ ) to allow the study to be powered for superiority and by setting an innovative long-term primary endpoint of clinical failure at 8 weeks post-therapy.

Traditional clinical trial endpoints occur soon after the end of treatment and cannot provide data on clinical failures or relapse rates occurring more than 2 or 3 weeks posttreatment. ${ }^{11,31}$ Consequently, they may not be long enough to reveal any clinically relevant differences in treatment regimens that emerge in the weeks following the completion of therapy. ${ }^{33}$ Indeed, it has been suggested that future clinical trials should be designed to assess longer-term endpoints. ${ }^{34,35}$ The suitability of an endpoint measured 8 weeks from the end of antibiotic treatment is supported by the fact that patients tend to relapse and exacerbations cluster such that there is a high risk of recurrent exacerbation in the 8 weeks after an initial exacerbation. ${ }^{33}$ In addition, in the MOSAIC study, the most significant differences between treatments in terms of time-totreatment failure, relapse, or requirement for further antibiotics occurred during the first 8 weeks after treatment. ${ }^{36}$

Patient selection is a recurring problem in published trials. It is now well established that underlying patient characteristics such as older age, higher exacerbation frequency, presence of concomitant cardiopulmonary disease, and a higher severity of underlying airway obstruction are key determinants of poor outcome in AECOPD. ${ }^{23,36,37}$ Exacerbations become more frequent and more severe as the underlying COPD progresses, but some patients have more exacerbations than others, which appears to be an independent susceptibility phenotype. ${ }^{38}$ Those who are particularly susceptible to exacerbations have worse health status and faster disease progression than infrequent exacerbators. ${ }^{38,39}$ One explanation might be that some patients remain at high risk for recurrent exacerbation in the 8-week period after initial exacerbation, ${ }^{33}$ possibly due to differing levels of ongoing lung and systemic inflammation in frequent and infrequent exacerbators. ${ }^{40}$ In most published antibiotic trials to date, patient populations are highly heterogeneous and may include relatively young and fit patients as well as those who are elderly and/or more severely ill. Indeed, there is even a lack of consensus on how to define patients' illnesses. While distinctions are made between acute exacerbation of chronic bronchitis (AECB) and AECOPD, COPD, and chronic bronchitis often coexist, are treated in parallel in the clinical setting, and most antibiotic intervention studies include patients with and without documented airflow obstruction. Enrollment of patients with AECB, mild COPD, or normal lung function will dilute the actual effect and increase the 
perceived efficacy of antibiotics due to high spontaneous rates of recovery. This makes it harder to show superiority of one antibiotic over another, ${ }^{11,31}$ as most of these patients would recover even without an antibiotic. As individuals at high risk of poor outcomes have high rates of failure and relapse, ${ }^{23,41}$ MAESTRAL is enriched with patients at risk of poor outcomes (ie, those $\geq 60$-years-old who have experienced $\geq 2$ exacerbations in the previous year, have moderate-to-severe COPD according to the GOLD spirometric criteria, ${ }^{16}$ and who are experiencing an Anthonisen type I exacerbation).

Patients with COPD are likely to be taking a range of medications to treat the exacerbation, the underlying COPD, and any concomitant illness. However, in many trials there is only limited evaluation of concurrent medication, the use of which could affect clinical outcome and create bias between antibiotic treatment groups. ${ }^{31}$ MAESTRAL tackles this issue by recording all concomitant medications for the full duration of the study. Physicians are instructed not to change patients' regular maintenance inhaled and oral COPD therapy during the study, but the need for any change in dosage or additional respiratory medication, such as bronchodilators and inhaled steroids, will be assessed.

Treatment of the current exacerbation often requires an antibiotic plus one or more other treatments, such as oral corticosteroids or an increased dose of a long-acting beta-agonist., ${ }^{9}$ To date, whether antibiotics are of benefit in moderate-to-severe exacerbations when a short course of corticosteroids is coadministered has been studied in very few large, well-designed trials. ${ }^{911,12}$ Because steroids can assist in the clinical resolution of an exacerbation, inconstant steroid treatment in the arms of an antibiotic trial can confound interpretation of the results. Mandating oral corticosteroids in all patients or making their use an exclusion criteria in this study are both inconsistent with current guidelines and make recruitment in a large study difficult. In MAESTRAL, the unique approach of stratifying patients for the concomitant use of oral corticosteroids to treat the current exacerbation is used to control for the use of concomitant corticosteroids. However, no inference on the clinical efficacy of steroid treatments can be drawn from this study because the allocation of steroids was not randomized.

Ensuring that patients most likely to be experiencing a bacterial (as opposed to, for instance, viral) exacerbation are enrolled in the trial is an important aspect of the MAESTRAL study design. Therefore, an Anthonisen type I exacerbation must be confirmed by the investigators. Where an AECOPD has a bacterial etiology, symptom resolution of that exacerbation will likely be influenced by bacterial eradication; the underlying mechanism is thought to be due to resolution of the inflammatory response to bacteria. ${ }^{42}$ By choosing patients with Anthonisen type 1 exacerbations, the study population is enriched with patients in whom bacteria play a significant role and who should benefit most from antibiotic therapy. Anthonisen type I exacerbations have been shown to have a high frequency of bacterial pathogen isolation. ${ }^{4}$

The MAESTRAL study uses recommended antibiotics, moxifloxacin and amoxicillin/clavulanic acid. Both drugs are commonly used for therapy of more severely ill patients with an AECOPD in most countries where the MAESTRAL trial is conducted, are recommended in a number of guidelines for this patient group, ${ }^{16,43}$ and have shown good efficacy in AECOPD. ${ }^{23,24}$ Furthermore there is evidence that fluoroquinolones, such as moxifloxacin, have resulted in better bacteriological eradication than some other drug classes. ${ }^{23}$ This was seen in the MOSAIC study, ${ }^{20}$ in which moxifloxacin was equivalent to standard comparators for short-term clinical outcomes, but was superior with respect to bacteriological eradication and resulted in fewer exacerbations in the 8 weeks following treatment than comparators..$^{20}$ MAESTRAL has built upon the design and outcomes of MOSAIC in a number of ways. As well as using the 8 -week endpoint, MAESTRAL enrolls patients identified in a post hoc analysis of MOSAIC data as having a high risk of relapse.

In the majority of noninferiority trials, sample size is calculated in order to detect a difference between treatment groups in failure rates of $<10 \%$. Conversely, in MAESTRAL, the high number of patients recruited was driven by a $6 \%$ margin for noninferiority, which is a much more rigorous definition. The use of a superiority protocol follows Food and Drug Administration guidance on trial design, which cautions that noninferiority trials have previously failed to show a benefit of one antibiotic over another. ${ }^{44} \mathrm{~A}$ further novel element in the design of MAESTRAL is the inclusion of AECB-SS and SGRQ questionnaires. Such patient-reported outcomes can provide important insights into clinical outcomes like symptom improvement and quality of life, as well as providing information that will enable the DRC to make informed decisions about the true clinical outcomes. Use of repeated spirometry measurements in MAESTRAL is also an innovative feature for an antibiotic trial, ensuring that only patients with moderate or severe COPD are enrolled and providing another means of monitoring response to treatment.

DRC members receive patient information in a blinded fashion for those who were clinical failures or had an indeterminate response. Through assessment of all documented clinical information and investigator decisions (although the DRC are 
blinded to clinical outcome assessments by investigators), the DRC is able to assess whether the patient is a true failure or relapse. This includes going back to investigators via the study managers (ie, in a blinded fashion) to challenge a decision. For example, when the clinical information suggests a clinical relapse, but antibiotic or oral steroid are not prescribed, it may be because the patient attended another clinic; whereas when clinical information suggests stability, but an antibiotic or steroid are prescribed, further questioning of the investigator could reveal that it was not prescribed for a relapse. The DRC also decides, based on prescribing habits, on steroid use that is a violation of protocol vs steroid use that is continued because of persistent symptoms, ie, clinical failure.

There are potential limitations to the design of MAESTRAL. The choice of clinical failure at 8 weeks posttherapy as the primary endpoint is based on the best evidence available, but may not prove to be the optimum duration potentially an even longer timescale might be desirable. However, it should also be noted that long-term follow-up can be subject to multiple confounders. Additionally, while steps have been taken to control for concomitant medications and oral corticosteroid use, stratification was limited to corticosteroid use and not undertaken for the use of long-acting bronchodilator and/or inhaled corticosteroids (though the inhaled medications were used at a stabilized dose).

Despite these limitations, it is anticipated that MAESTRAL will provide further evidence as to the most appropriate study design for trials of antibiotics in AECOPD, and it should also provide some information about the extent of the relative roles of antibiotics and systemic corticosteroids in the treatment of AECOPD. Irrespective of whether noninferiority or superiority is achieved, the results should add further to the debate on optimal trial design in this condition. It is hoped that MAESTRAL may also help to inform guidelines and allow physicians to optimize clinical outcomes and reduce AECOPD-related health care costs through appropriate patient profiling and antibiotic choice.

\section{Acknowledgments}

This study was funded by Bayer HealthCare Pharmaceuticals. Highfield Communication Consultancy (funded by Bayer HealthCare Pharmaceuticals) provided editorial assistance in the preparation of this manuscript. RW was supported by the NIHR Respiratory Disease Biomedical Research Unit.

\section{Disclosure}

RW, SS, AA, and MM have acted as consultants and received honoraria as speakers in scientific meetings or courses organized by the study sponsor. PA and DH are employees of Bayer HealthCare and Bayer HealthCare Pharmaceuticals (France and USA, respectively). MT and GF are employees of Bayer Inc, Canada.

\section{References}

1. Donaldson GC, Seemungal TA, BhowmikA, Wedzicha JA. Relationship between exacerbation frequency and lung function decline in chronic obstructive pulmonary disease. Thorax. 2002;57(10):847-852.

2. Kanner RE, Anthonisen NR, Connett JE; for the Lung Health Study Research Group. Lower respiratory illnesses promote FEV(1) decline in current smokers but not ex-smokers with mild chronic obstructive pulmonary disease: results from the lung health study. Am J Respir Crit Care Med. 2001;164(3):358-364.

3. Martinez FJ, Han MK, Flaherty K, Curtis J. Role of infection and antimicrobial therapy in acute exacerbations of chronic obstructive pulmonary disease. Expert Rev Anti Infect Ther. 2006;4(1):101-124.

4. Soler-Cataluña JJ, Martínez-García MA, Román Sánchez P, et al. Severe acute exacerbations and mortality in patients with chronic obstructive pulmonary disease. Thorax. 2005;60(11):925-931.

5. Anzueto A, Sethi S, Martinez FJ. Exacerbations of chronic obstructive pulmonary disease. Proc Am Thorac Soc. 2007;4(7):554-564.

6. Ramsey SD, Hobbs FD. Chronic obstructive pulmonary disease, risk factors, and outcome trials: comparisons with cardiovascular disease. Proc Am Thorac Soc. 2006;(7)3:635-640.

7. Niewoehner DE. The impact of severe exacerbations on quality of life and the clinical course of chronic obstructive pulmonary disease. $\mathrm{Am}$ J Med. 2006;119(10 Suppl 1):S38-S45.

8. Llor C, Molina J, Naberan K, Cots JM, Ros F, Miravitlles M; for the EVOCA study group. Exacerbations worsen the quality of life of chronic obstructive pulmonary disease patients in primary healthcare. Int J Clin Pract. 2008;62(4):585-592.

9. Wilkinson T, Wedzicha JA. Strategies for improving outcomes of COPD exacerbations. Int J Chron Obstruct Pulmon Dis. 2006;1(3): 335-342.

10. Dalal AA, Christensen L, Liu F, Riedel AA. Direct costs of chronic obstructive pulmonary disease among managed care patients. Int J Chron Obstruct Pulmon Dis. 2010;5:341-349.

11. Sethi S, Murphy TF. Acute exacerbations of chronic bronchitis: new developments concerning microbiology and pathophysiology - impact on approaches to risk stratification and therapy. Infect Dis Clin North Am. 2004;18(4):861-882.

12. Daniels JM, Snijders D, de Graaff CS, Vlaspolder F, Jansen HM, Boersma WG. Antibiotics in addition to systemic corticosteroids for acute exacerbations of chronic obstructive pulmonary disease. $\mathrm{Am}$ J Respir Crit Care Med. 2010;181(2):150-157.

13. Aaron SD, Vandemheen KL, Hebert P, et al. Outpatient oral prednisone after emergency treatment of chronic obstructive pulmonary disease. N Engl J Med. 2003;348(26):2618-2625.

14. Adams SG, Melo J, Luther M, Anzueto A. Antibiotics are associated with lower relapse rates in outpatients with acute exacerbations of COPD. Chest. 2000;117(5):1345-1352.

15. Woodhead M, Blasi F, Ewig S; European Respiratory Society; European Society of Clinical Microbiology and Infectious Diseases. Guidelines for the management of adult lower respiratory tract infections. Eur Resp J. 2005;26(6):1138-1180.

16. The Global Initiative for Chronic Obstructive Lung Disease (GOLD). Global Strategy for Diagnosis, Management, and Prevention of COPD. Available at: http://goldcopd.com. Accessed April 4, 2011.

17. Dever LL, Shashikumar K, Johanson WG Jr. Antibiotics in the treatment of acute exacerbations of chronic bronchitis. Expert Opin Investig Drugs. 2002;11(7):911-925.

18. Sethi S. Antibiotics in acute exacerbations of chronic bronchitis. Expert Rev Anti Infect Ther. 2010;8(4):405-417.

19. Miravitlles M, Torres A. No more equivalence trials for antibiotics in exacerbations of COPD, please. Chest. 2004;125(3):811-813. 
20. Wilson R, Allegra L, Huchon G; for the MOSAIC Study Group. Short-term and long-term outcomes of moxifloxacin compared to standard antibiotic treatment in acute exacerbations of chronic bronchitis. Chest. 2004;125(3):953-964.

21. Anthonisen NR, Maanfreda J, Warren CP, Hershfield ES, Harding GK, Nelson NA. Antibiotic therapy in exacerbations of chronic obstructive pulmonary disease. Ann Intern Med. 1987;106(2):196-204.

22. Clinical Laboratory Standards Institute. Methods for Dilution Antimicrobial Susceptibility Tests for Bacteria That Grow Aerobically; Approved Standards - Eighth Edition. CLSI document M07-A8 Wayne, PA, 2009.

23. Miravitlles M. Moxifloxacin in the management of exacerbations of chronic bronchitis and COPD. Int J Chron Obstruct Pulmon Dis. 2007; 2(3):191-204.

24. Sethi S, Breton J, Wynne B. Efficacy and safety of pharmacokinetically enhanced amoxicillin-clavulanate at 2000/125 milligrams twice daily for 5 days versus amoxicillin-clavulanate at 875/125 milligrams twice daily for 7 days in the treatment of acute exacerbations of chronic bronchitis. Antimicrob Agents Chemother. 2005;49(1):153-160.

25. White AR, Kaye C, Poupard J, et al. Augmentin ${ }^{\circledR}$ (amoxicillin/ clavulanate) in the treatment of community-acquired respiratory tract infection: a review of the continuing development of an innovative antimicrobial agent. J Antimicrob Chemother. 2004;53(Suppl 1):i3-i20.

26. Summary of Product Characteristics. Avelox $400 \mathrm{mg}$ film-coated tablets. Bayer Schering Pharma (Bayer UK). Available at: http://www. medicines.org.uk/EMC/medicine/11841/SPC/Avelox+400+mg+filmcoated+tablets. Accessed April 4, 2011.

27. Jones PW, Quirk FH, Baveystock CM. The St George's Respiratory Questionnaire. Respir Med. 1991;85 (Supp1 B):25-31; discussion 33-37.

28. Jones PW. Activity limitation and quality of life in COPD. COPD. 2007; 4(3):273-278.

29. Jones PW. The Acute Exacerbation of COPD Symptom Scale (AECB-SS). J COPD Management. 2006; Quarter 2, article 3.

30. Food and Drug Administration. Guidance for Industry Non-Inferiority Clinical Trials Additional. 2010. Available from: http://www.fda gov/downloads/Drugs/GuidanceComplianceRegulatoryInformation/ Guidances/UCM202140.pdf. Accessed April 4, 2011.

31. Patel A, Wilson R. Newer fluoroquinolones in the treatment of acute exacerbations of COPD. Int J Chron Obstruct Pulmon Dis. 2006;1(3): 243-250.

32. Siddiqi A, Sethi S. Optimizing antibiotic selection in treating COPD exacerbations. Int J Chron Obstruc J Pulmon Dis. 2008;3(1):31-44.
33. Hurst JR, Donaldson GC, Quint JK, et al. Temporal clustering of exacerbations in chronic obstructive pulmonary disease. Am J Respir Crit Care Med. 2009;179(5):369-374.

34. Anzueto A, Rizzo JA, Grossman RF. The infection-free interval: its use in evaluating antimicrobial treatment of acute exacerbation of chronic bronchitis. Clin Infect Dis. 1999;28(6):1344-1345.

35. Chodosh S. Clinical significance of the infection-free interval in the management of acute bacterial exacerbations of chronic bronchitis. Chest. 2005;127(6):2231-2236.

36. Wilson R, Jones P, Schaberg T; for the MOSAIC Study Group. Antibiotic treatment and factors influencing short and long term outcomes of acute exacerbations of chronic bronchitis. Thorax. 2006;61(4): $337-342$.

37. Miravitlles M, Guerrero T, Mayordomo C, et al. Factors associated with increased risk of exacerbation and hospital admission in a cohort of ambulatory COPD patients: a multiple logistic regression analysis. The EOLO Study Group. Respiration. 2000;67(5):491-492.

38. Hurst JR, Vestbo J, Anzueto A; for the Evaluation of COPD Longitudinally to Identify Predictive Surrogate Endpoints (ECLIPSE) Investigators. Susceptibility to exacerbation in chronic obstructive pulmonary disease. N Engl J Med. 2010;363(12):1128-1138.

39. Wedzicha JA, Seemungal TA. COPD exacerbations: defining their cause and prevention. Lancet. 2007;370(9589):786-796.

40. Perera WR, Hurst JR, Wilkinson TM, et al. Inflammatory changes, recovery and recurrence at COPD exacerbation. Eur Respir J. 2007; 29(3):527-534.

41. Dewan NA, Rafique S, Kanwar B, et al. Acute exacerbation of COPD: factors associated with poor treatment outcome. Chest. 2000;117(3): 662-671.

42. White AJ, Gompertz S, Bayley DL, et al. Resolution of bronchial inflammation is related to bacterial eradication following treatment of exacerbations of chronic bronchitis. Thorax. 2003;58(8):680-685.

43. O'Donnell DE, Aaron S, Bourbeau J, et al. Canadian Thoracic Society recommendations for management of chronic obstructive pulmonary disease - 2007 update. Can Respir J. 2007;14(Suppl B):5B-32B.

44. Food and Drug Administration. Guidance for Industry. Acute Bacterial Exacerbations of Chronic Bronchitis in Patients with Chronic Obstructive Pulmonary Disease: Developing Antimicrobial Drugs for Treatment. 2008. Available at: http://www.fda.gov/downloads/Drugs/ GuidanceComplianceRegulatoryInformation/Guidances/ucm070935. pdf. Accessed April 4, 2011. 


\section{Supplementary material}

\section{Supplementary section I: Full list of exclusion criteria}

I. Known hypersensitivity to quinolones, beta-lactams, or to any of the excipients of the study drugs.

2. Pregnant or breastfeeding women (women of childbearing potential, based on investigator assessment, must have negative urinary pregnancy test).

3. Congenital or acquired QT prolongation.

4. Clinically relevant bradycardia.

5. Clinically relevant heart failure with reduced left-ventricular ejection fraction.

6. Previous history of symptomatic arrhythmias.

7. Taking QT prolonging drugs, for example Class la or III anti-arrhythmic agents (eg, quinidine, procainamide, amiodarone, sotalol), neuroleptics (eg, phenothiazines, pimozide, sertindole, haloperidol, sultopride), tricyclic antidepressants, certain antihistaminics (eg, terfenadine, astemizole, mizolastine), certain antimicrobials (sparfloxacin; erythromycin IV; pentamidine; antimalarials, particularly halofantrine), or other QT prolonging drugs (eg, cisapride, intravenous vincamine, bepridil, and diphemanil).

8. Uncontrolled electrolyte disturbances, particularly uncorrected hypokalaemia.

9. History of hereditary problems of galactose intolerance, the Lapp lactase deficiency, or glucose-galactose malabsorption.

10. History of a tendon disease/disorder.

II. History of liver dysfunction (Child-Pugh C), including elevated transaminase levels (alanine aminotransferase and/or aspartate aminotransferase $>5$ times the upper limit of normal).

12. Severe renal impairment with glomerular filtration rate of $<30 \mathrm{~mL} / \mathrm{min}$.

13. Neutropenia (neutrophil count $<1000 / \mathrm{mm}^{3}$ ) caused by immunosuppressive therapy or malignancy.

14. AIDS (CD4 count of $<200 / \mathrm{mm}^{3}$ ), or HIV positive and receiving highly active anti-retroviral therapy (testing for HIV is not mandatory).

15. Chronic asthma (>15\% reversibility or at least $200 \mathrm{~mL}$ ), bronchial carcinoma, active pulmonary tuberculosis, known diffuse bronchiectasis, cystic fibrosis, or pneumonia (a chest $\mathrm{X}$-ray is not mandatory).

16. History of chronic colonization of pathogenic organisms resistant to moxifloxacin and/or amoxicillin/clavulanic acid (eg, Pseudomonas aeruginosa, meticillin-resistant Staphylococcus aureus).

17. Receiving long-term ( $>4$ consecutive weeks) systemic corticosteroid treatment ( $>10 \mathrm{mg} /$ day of prednisolone or equivalent).

18. Received short course of systemic corticosteroid treatment within 30 days prior to enrollment.

19. Unable to take oral medication.

20. Life expectancy of less than 6 months.

21 . Receiving systemic antibacterial therapy within 30 days prior to study enrollment.

22. Requiring concomitant systemic antibacterial agents.

23. Use of any investigational drug or device within 30 days of screening, or previously enrolled in this study.

24. Requiring home ventilatory support (patients requiring home/portable oxygen therapy or continuous positive airway pressure for sleep apnea are not excluded) and/or those who have a tracheotomy in situ.

25. History of liver function disorders following previous treatment with amoxicillin/clavulanic acid.

26. Receiving disulfiram therapy.

Abbreviations: AIDS, acquired immune deficiency syndrome; HIV, human immunodeficiency virus.

\section{Supplementary section 2: Protocol for stratification according to steroid use and geographical region}

\section{Steroid use}

Prior to randomization, patients are stratified according to steroid usage. Stratum I patients were defined as having had coadministration of systemic corticosteroids for the current AECOPD, ie:

- were not on long-term low-dose systemic corticosteroid treatment; and

- had received treatment with systemic corticosteroids ( $\geq 50 \mathrm{mg}$ over 10 days) for the current AECOPD.

Stratum 2 patients were defined as having had no coadministration of systemic corticosteroids for the current AECOPD, ie:

- were on long-term low-dose systemic corticosteroid treatment which remained stable or increased minimally during the study treatment

(cumulative dose $<100 \mathrm{mg}$ over 10 days) for the current AECOPD; or

- had received systemic corticosteroids for the current AECOPD but at a cumulative dose of $<50 \mathrm{mg}$ over 10 days.

\section{Geographical regions}

For the primary analysis, the following geographical regions will be included as separate strata:

- Asia Pacific: Australia, China, Hong Kong, Indonesia, Pakistan, the Philippines, Thailand

- Europe I: Belgium, Germany, Ireland, Latvia, Lithuania, the Netherlands, Switzerland, UK

- Europe 2 and South Africa: Andorra, Croatia, Czech Republic, Greece, Italy, Portugal, South Africa, Spain

- Latin America and Canada: Argentina, Mexico, Peru, Brazil, Colombia, Chile, Canada.

The ratio of the sample size of the largest region compared with the smallest region should not be greater than 2:I.

Abbreviation: AECOPD, acute exacerbations of chronic obstructive pulmonary disease. 
Supplementary section 3: Clinical and bacteriological response categories

Clinical response definitions

- Clinical improvement: signs and symptoms are improving and treatment continues (this category is only available during antibiotic therapy).

- Clinical failure: exacerbation symptoms have not improved or have worsened such that additional or alternate systemic antimicrobial and/ or systemic corticosteroid therapy is required.

- Indeterminate: clinical response cannot be determined.

- Clinical cure: resolution or improvement in the signs and symptoms of the exacerbation such that additional or alternate systemic antimicrobial and/or systemic corticosteroid therapy is not required.

- Continued clinical cure: continued absence of acute signs and symptoms related to the infection such that additional or alternate therapy (systemic antimicrobial and/or systemic corticosteroid) is not required.

- Clinical relapse: reappearance of the signs and symptoms of infection such that re-institution of therapy (systemic antimicrobial and/or systemic corticosteroid) is required; for the analysis, this will be considered clinical failure at the 4 weeks post-therapy and 8 weeks post-therapy visits.

\section{Bacteriological response definitions}

- Bacteriological eradication without superinfection or reinfection: initial causative pathogen(s) absent, no new pathogen isolated after start of study.

- Presumed eradication: absence of appropriate culture material for evaluation because subject has clinically improved on therapy.

- Persistence: initial causative pathogen(s) still present.

- Presumed persistence: absence of appropriate culture material for evaluation, in a subject who has not clinically improved on therapy.

- Bacteriological eradication with superinfection: initial causative pathogen(s) absent, a new pathogen isolated during treatment.

- Bacteriological eradication with reinfection: initial causative pathogen(s) absent, a new pathogen isolated after end of treatment.

- Eradication with recurrence: original causative organism absent at end of therapy, but reappearance of the same organism at or before 8 weeks post-therapy.

- Continued eradication: the causative organism(s) is absent at this time point.

- Continued presumed eradication: the absence of appropriate culture material for evaluation because the patients have clinically improved.

- Indeterminate: bacterial response to the study drug not evaluable.

\section{Supplementary section 4: Subpopulations of interest}

- Males, females

- $<65$ years of age, and elderly patients ( $\geq 65$ years)

- 2, 3, and $\geq 4$ previous AECB episodes in the last 12 months

- Active smokers at study start

- Cardiopulmonary disease (as defined by the MedDRA terms)

- Previous respiratory failure

- Patients receiving (stratum I) or not receiving (stratum 2) coadministration of systemic steroid administration

- Patients receiving coadministration of systemic steroid administration, irrespective of stratum allocation

- $\mathrm{FEV}$, at enrollment according to the following categories: $\mathrm{FEV}_{1}<30 \%$ of predicted, $30 \% \leq \mathrm{FEV},<60 \%$ of predicted, and $\mathrm{FEV}_{1} \geq 60 \%$ of predicted

- Previous systemic antimicrobial use in the last 90 days before study start (for any reason)

- Patients whose last exacerbation was $\geq 63$ days before study start

- Patients whose last exacerbation was $<6$ months before study start

- Patients with bacteria isolated from sputum at baseline

Abbreviations: $A E C B$, acute exacerbation of chronic bronchitis; $\mathrm{FEV}_{1}$, forced expiratory volume in I second; MedDRA, Medical Dictionary for Regulatory Activities.
International Journal of COPD

\section{Publish your work in this journal}

The International Journal of COPD is an international, peer-reviewed journal of therapeutics and pharmacology focusing on concise rapid reporting of clinical studies and reviews in COPD. Special focus is given to the pathophysiological processes underlying the disease, intervention programs, patient focused education, and self management protocols.

\section{Dovepress}

This journal is indexed on PubMed Central, MedLine and CAS. The manuscript management system is completely online and includes a very quick and fair peer-review system, which is all easy to use. Visit http://www.dovepress.com/testimonials.php to read real quotes from published authors. 